\title{
Synthesis of Hydrophilic Derivative Surfactants From Algae-Derived Unsaponifiable Lipids
}

\author{
Gina Fioroni, Rui Katahira, Stefanie Van Wychen, Steven M. Rowland, Earl D. Christensen, \\ Tao Dong, Philip T. Pienkos and Lieve M. L. Laurens*
}

National Renewable Energy Laboratory, Golden, CO, United States

In the context of decarbonizing the economy, the utilization of biologically sourced feedstocks to produce replacements for petroleum-derived materials is becoming more urgent. Improving renewable biomass production and utilization is imperative for commercializing future biorefineries. Algae-derived biomass is a particularly promising feedstock thanks to its attractive oil content and composition; specifically, the high-value

OPEN ACCESS

Edited by:

Edwin Zondervan,

University of Twente, Netherlands

Reviewed by:

Meei Mei N. Gui,

Queen's University Belfast,

United Kingdom

Matthijs Ruitenbeek,

Dow Benelux, Netherlands

Manishkumar S. Tiwari,

SKVM's NMIMS University, India

Hitesh Pawar,

Institute of Chemical Technology, India

*Correspondence:

Lieve M. L. Laurens

Lieve.Laurens@nrel.gov

Specialty section:

This article was submitted to

Sustainable Process Engineering,

a section of the journal

Frontiers in Chemical Engineering

Received: 31 August 2021 Accepted: 17 December 2021

Published: 11 January 2022

Citation:

Fioroni G, Katahira R, Van Wychen S, Rowland SM, Christensen ED, Dong T, Pienkos PT and Laurens LML (2022)

Synthesis of Hydrophilic Derivative

Surfactants From Algae-Derived

Unsaponifiable Lipids.

Front. Chem. Eng. 3:768382.

doi: 10.3389/fceng.2021.768382 products in the unsaponifiable lipids have not been included in a conversion process. Here we demonstrate surfactant synthesis from a complex oil fraction as the hydrophobic donor moieties, yielding products that are similar to commercially available surfactants such as the linear alkyl benzene sulfonates. Unsaponifiable lipids extracted from algae were derivatized to non-ionic surfactants using a green chemical synthesis route based on a double esterification with succinic acid and polyethylene glycol. The in-depth molecular and structural surfactant characterization is included and indicates that the resulting properties fall between those of pure cholesterol and phytol used as surrogates for the reaction synthesis demonstration. This is the first demonstration of an effective and potentially high-value synthesis of functional surfactants with properties that can be tailored based on the relative composition of the resulting hydrocarbon alcohol components in the mixture. This novel green chemistry synthesis approach provides a route to high-value product synthesis from algae.

Keywords: oleochemicals, sterols, phytol, biobased surfactants, algae

\section{INTRODUCTION}

A recent drive to decarbonize the economy has seen a push, primarily driven by consumers, to replace petrochemical surfactants and detergents with biobased alternatives. Similar to the growth of the petrochemical industry, the oleochemicals industry has seen tremendous growth in the use of biobased oils as the donor for the hydrophobic moiety in renewable surfactants. In particular, commercially available linear alkyl benzene sulfonate surfactants represent a large proportion of the non-ionic surfactants market, with diverse applications that are currently dominated by petroleumderived products (Kjellin and Johansson, 2010; Pleissner et al., 2014; Laurens et al., 2017). To make a market impact with alternative biobased non-ionic surfactants, it is critical that both the surfactant properties and molecular composition approximate the target application. Furthermore, the biological feedstock for the hydrophobic moieties of the surfactants should be sustainably sourced, chemically well-characterized, and abundantly available. One such feedstock is algal biomass, derived from single-celled photosynthetic organisms, whose chemical composition can 
be optimized and tuned to provide a sustainable source of oleochemicals (Koutinas et al., 2014; Laurens et al., 2017).

A fraction of algae oils-the unsaponifiable lipids-comprise high-value oleochemical precursors that can be isolated but have not yet been exploited in conversion pathways to biofuels and biobased products. Removing the unsaponifiable fraction of the lipids, including the pigments, sterols, phytol, and other products, not only provides a feedstock for biobased product development, but also aids with the downstream catalytic upgrading of the fatty acid fraction to renewable diesel and jet fuels of the future (Kruger et al., 2017). Sterols and phytol are present in algal oil and are not ideal fuel precursor molecules, as they require significant amounts of hydrogen for hydrotreating and cracking to convert to hydrocarbons of the appropriate chain length for renewable diesels or jet fuel (Davis et al., 2014; Wagner et al., 2014; Dong et al., 2015; Laurens et al., 2015).

Renewable surfactants can be synthesized through ethoxylation of the alcohol group in phytol and the free sterols. This process can be relatively toxic and is not straightforward because steric hindrance reduces the reactivity of these secondary hydroxyl groups compared to primary alcohols (Johansson and Svensson, 2001; Folmer, 2003). An alternative, novel synthesis route has been developed that uses a green chemical approach based on consecutive double esterification of a hydrophobic moiety with at least an accessible hydroxyl donor, with succinic acid and polyethylene glycol to create non-ionic surfactants (Lim and Chung, 2011). The hydrophobic donor molecules can be diverse, ranging from fatty acids to complex sterols. The polyethylene glycol chain that is attached to the sterol can be varied in chain length to adjust the final surfactant properties.

Unsaponifiable lipids, including sterols, are ideally suited to be the hydrophobic donors to a biobased surfactant synthesis pathway. The unsaponifiable lipids in algae are a complex mixture, with most of the products assigned to the sterol or phytol class of compounds, both ideal hydrophobic constituents for potentially high-value bio-derived surfactants. The unsaponifiables are usually isolated after a strong alkaline treatment, which saponifies all the fatty-acid-derived lipids, rendering the fatty acids water-soluble, and phytol and sterols can either be purified for further use or used as a mixture with the phytol fraction retained for derivatization of the hydroxyl groups to form amphiphilic, non-ionic surfactants. Surfactants produced from sterols can be utilized in many different commercial products, including cosmetics and soaps (Folmer, 2003; Kjellin and Johansson, 2010; Urbin et al., 2014).

Previously reported mass spectral analysis indicates that there are over 20 different sterols identified in different species of algae (Ryckebosch et al., 2014; Ahmed et al., 2015). The wide range of sterols present indicates significant molecular and metabolic flexibility of algae in their native isoprenoid metabolism and opens a wide range of coproduct development options for the valorization of sterols, though the complexity of the mixtures ensures difficulties in developing coproducts based on pure compounds. The compounds we identified fall within two major classes: 28-carbon 4-desmethylsterols and 29-carbon 4desmethylsterols. Within the 28-carbon group, the spectra either more closely resemble campesterol (molecular weight 472) or ergosterol (molecular weight 468). Within the 29-carbon group, the spectra could be grouped into compounds with a molecular weight of 484 (e.g., stigmasterol, fucosterol, $\Delta 5$-avenasterol) or those with a molecular weight of 486 (sitosterol or $\Delta 7$ stigmastenol). This complexity of sterols in algal lipids should not impact the value of surfactant feedstocks when used without isolating single components, as long as the surfactant product specifications are met since many commercially available surfactants (e.g., linear alkyl benzene sulfonates) are often mixtures of compounds (Ashford, 2011).

The objective of the work described here is to demonstrate the effective synthesis and in-depth characterization of novel biobased surfactants from a complex mixture of hydroxylcontaining unsaponifiable lipids extracted from algal biomass in a process-relevant manner. To our knowledge, this is the first demonstration of the use of unsaponifiable lipids from photosynthetic algae as the feedstock for the synthesis of biobased surfactants.

\section{MATERIALS AND METHODS}

\section{Biomass and Chemicals}

Unless stated otherwise, all chemicals were purchased from Sigma Aldrich at purities of $95 \%$ or higher; specifically, 95\% pure cholesterol (C75209); 97\% mixture of phytol isomers, 1:2 cis: trans isomer mixed phytol (139912); polyethylene glycol (PEG) with average molecular weight of 1,000 Da (8074881000); and succinic anhydride (8006830100).

Algal biomass from Scenedesmus acutus (LRB-AP 0401) was kindly provided by Dr. J. McGowen at the Arizona Center for Algae Technology and Innovation (AzCATI) at Arizona State University. In brief, biomass was cultivated in a controlled fashion in outdoor flat-panel (650-L) photobioreactors in nitrate-deplete cultivation media. Cultivation time after reaching nutrient-deplete conditions depended on final target biomass composition desired-in this case 6 days for lipid accumulation under nutrient-deplete conditions to reach the targets of high carbohydrate and lipid content (Laurens et al., 2014). Harvesting the biomass was accomplished using centrifugation (Alfa Laval, Lund, Sweden), and the material was frozen until needed. The biomass composition consisted of $30.8 \pm 0.4 \%$ lipids (measured as fatty acid methyl esters through direct transesterification) and $44.2 \pm 1.4 \%$ total carbohydrates, measured as described in Laurens et al. (2014).

\section{Lipid and Unsaponifiables Extraction}

For crude lipid extraction from algal biomass, triplicate samples of $20 \mathrm{ml}$ of $10 \%$ (w/v) algal slurry of S. acutus biomass, acidified with sulfuric acid to $1 \% \mathrm{w} / \mathrm{v}$, was added to three $50-\mathrm{ml}$ glass tubes, for a total of $60 \mathrm{ml}$ of slurry extracted. Tubes were capped after adding a stir bar, and the pretreatment program was run at $150^{\circ} \mathrm{C}$ for $15 \mathrm{~min}$. After pretreatment, the slurry was allowed to cool before being transferred to an Erlenmeyer flask for extraction. Hexanes were added to the slurry in a 1:1 v/v ratio and allowed to extract overnight at room temperature. The hexane was removed 
from the slurry and placed into a separate vessel for drying. Two more hexane extractions were performed, each for $1 \mathrm{~h}$. The hexane extracts were dried down under nitrogen and a final weight of the oil was recorded: approximately $1.3 \mathrm{~g}$ combined from triplicate pretreatment samples.

To isolate the unsaponifiables, the resulting algal oil was saponified following a standard AOCS method, minimally modified (American Oil Chemists' Society, 2009). In brief, approximately $1 \mathrm{~g}$ of oil was transferred into a glass tube with $1.5 \mathrm{ml}$ of $\mathrm{KOH}(60 \mathrm{~g} / 40 \mathrm{ml})$ and $12.5 \mathrm{ml}$ of ethanol. The mixture was heated at $80^{\circ} \mathrm{C}$ for $90 \mathrm{~min}$ in a hot block, mixing every $10 \mathrm{~min}$, and contents were transferred to a separatory funnel while still warm. The mixture was first washed with $18.75 \mathrm{ml}$ of diethyl ether and $18.75 \mathrm{ml}$ of deionized water. The aqueous layer was then drained and saved. The organic layer was rinsed again with $12.5 \mathrm{ml}$ of deionized water. The aqueous phases were combined, and the organic phase was collected in a separate vessel. The combined aqueous phases were placed back in a separatory funnel and washed with $18.75 \mathrm{ml}$ of diethyl ether to ensure complete extraction; the aqueous phase was then discarded. The organic phases were combined and washed with $7.5 \mathrm{ml}$ of $0.5-\mathrm{M} \mathrm{KOH}$ to remove any additional free fatty acids. The $\mathrm{KOH}$ layer was then drained off, and the organic phase was washed with $7.5 \mathrm{ml}$ of deionized water. The $\mathrm{KOH}$ and water washes were repeated two additional times. The organic layer was then washed with approximately $100 \mathrm{ml}$ of deionized water in $10-\mathrm{ml}$ increments to ensure all salts were removed. The organic layer was dried down under nitrogen, and a gravimetric yield was recorded as total unsaponifiables, which were then used in the surfactant synthesis methods as described below. An oil yield of $22 \%$ (on a dry weight biomass basis) was obtained from the dilute acid pretreatment described above. The unsaponifiables fraction was $4.5 \%$ of the extracted oil. This fraction was dried under nitrogen stored in the freezer $\left(-20^{\circ} \mathrm{C}\right)$ until use.

\section{Gas Chromatography of Unsaponifiables}

The extracted unsaponifiables were brought up in the appropriate amount of chloroform prior to derivatization for gas chromatography (GC). A total of $75 \mu \mathrm{l}$ of the dissolved extract was transferred to a $300-\mu \mathrm{l}$ insert vial, and $25 \mu \mathrm{l}$ of $1: 1(\mathrm{v} / \mathrm{v})$ BSTFA (1\% TMCS):pyridine was added to derivatize the extracted sterols. Standards were also prepared by adding $25 \mu \mathrm{l}$ of 1:1 (v/v) BSTFA (1\% TMCS):pyridine and $5 \mu \mathrm{l}$ of $\sim 1 \mathrm{mg} / \mathrm{ml} 5 \mathrm{a}-$ cholestane (internal standard). The internal standard was also spiked into the samples before they were analyzed per the methods described above, to determine extraction efficiency.

All samples were analyzed by GC-flame ionization detection and GC-mass spectrometry (MS) (for identification only). Analysis was performed using an Agilent 6890N gas chromatograph with an Agilent flame ionization detector (Agilent Technologies Inc., Palo Alto, CA). Using a pulsed (hold time $0.2 \mathrm{~min}, 60 \mathrm{psi}$ ) splitless injection, $1 \mu \mathrm{l}$ was introduced onto a $30 \mathrm{~m} \times 0.25 \mathrm{~mm}$ internal diameter (ID), $0.25-\mu \mathrm{m}$ film thickness HP-5MS capillary column (J\&W Scientific Inc., Folsom, CA) at $350^{\circ} \mathrm{C}$. The helium flow was kept constant at $1 \mathrm{ml} / \mathrm{min}$ with an oven gradient as follows: The initial column temperature of $50^{\circ} \mathrm{C}$ was held for $3 \mathrm{~min}$ and then increased by $10^{\circ} \mathrm{C} / \mathrm{min}$ to $270^{\circ} \mathrm{C}$ for $10 \mathrm{~min}$, after which the temperature was increased to $325^{\circ} \mathrm{C}$ at $20^{\circ} \mathrm{C} / \mathrm{min}$ and held for $15 \mathrm{~min}$ for a total run time of $52.75 \mathrm{~min}$.

\section{Synthesis of Carboxyethyl Derivatives of Cholesterol, Phytol, and Unsaponifiables}

The general synthesis scheme for hydrophilic derivatives of sterols was carried out following a previously published procedure (Figure 1) (Lim and Chung, 2011). Cholesterol $(5 \mathrm{~g})$, succinic anhydride (1.5 eq., $1.94 \mathrm{~g})$, and dimethylaminopyridine (DMAP) (0.02 eq., $0.031 \mathrm{~g})$ were dissolved in $25 \mathrm{ml}$ of toluene. The solution was stirred and refluxed until complete by thin-layer chromatography (about $6 \mathrm{~h}$ ). The solution was then cooled, and $40 \mathrm{ml}$ of hexane was added to precipitate the product. The resulting solid was removed by vacuum filtration and recrystallized in ethanol $(20 \mathrm{ml})$ overnight in the freezer to afford pure carboxyethyl derivative cholesterol-succinic anhydride (SA) (6.18 g, 98.4\% yield). PhytolSA was prepared in the same manner as cholesterol-SA. However, the desired pure phytol-SA was recovered as a very viscous white liquid from the liquid fraction of the mixture, with the solid portion of the undesired byproducts from the reaction being filtered off (6.2439 g, 94.4\% yield). Mixed-algae unsaponifiablesSA was prepared in the same manner as cholesterol-SA. Confirmation of the synthesis products by proton nuclear magnetic resonance $\left({ }^{1} \mathrm{H}-\mathrm{NMR}\right)$ revealed a mixed product; however, the characteristic carbonyl protons were present, indicating that the succinic anhydride had successfully been added. This product was used crude in the next step. All calculations and yields were based off of cholesterol as the molecular weight.

\section{Synthesis of Carboxyethyl Polyethylene Glycol Derivatives of Cholesterol, Phytol, and Unsaponifiables}

To synthesize cholesterol-SA-PEG, polyethylene glycol (PEG 1000, 3 eq., $7.9015 \mathrm{~g}$ ), dicyclohexylcarbodiimide (DCC) (1.25 eq., $0.5306 \mathrm{~g})$, and DMAP (0.06 eq., $0.02 \mathrm{~g}$ ) were dissolved in $40 \mathrm{ml}$ of dichloromethane in a round-bottom flask. Cholesterol-SA (1.0313 g) dissolved in $40 \mathrm{ml}$ of dichloromethane was added dropwise. The appearance of the precipitated dicyclohexylurea byproduct was noted after about $5 \mathrm{ml}$ of the cholesterol-SA solution was added. After completion of the reaction, the dicyclohexylurea was removed by filtration and the filtrate was washed with $100 \mathrm{ml}$ of a $5 \%$ $\mathrm{NaCl}_{2}$ solution. The solution was evaporated under vacuum to produce a white solid. The crude product was dissolved in $20 \mathrm{ml}$ of $3: 1$ hexane:isopropanol (IPA) at $40^{\circ} \mathrm{C}$ and then stored in the freezer overnight to recrystallize. Pure cholesterol-SAPEG was recovered via vacuum filtration $(2.839 \mathrm{~g}, 90.5 \%$ yield; overall yield for two steps: $89.1 \%$ ). Phytol-SA-PEG was prepared in the same manner as cholesterol 1,000, with an overall yield for two steps of $99.9 \%$. Unsaponifiables-SA-PEG was prepared in the same manner as cholesterol-SA-PEG. The unsaponifiables-SA-PEG was used without further 


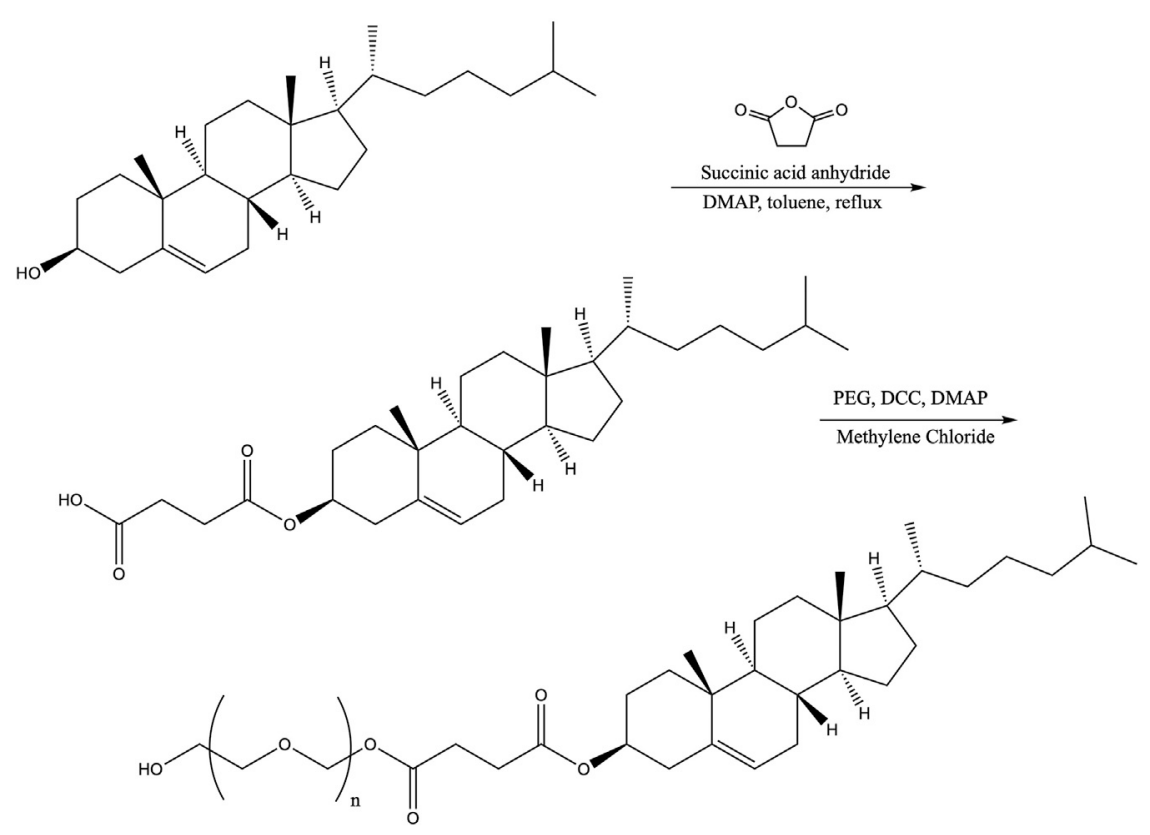

FIGURE 1 | Synthesis reaction scheme used in this work for carboxyethyl-PEG derivatives of hydroxylated hydrophobic moiety donors (shown here with cholesterol and succinic anhydride).

purification. Overall yield for two steps based on cholesterol molecular weight was $65.1 \%$.

\section{Characterization by ${ }^{1} \mathrm{H}-\mathrm{NMR}$ and ${ }^{13} \mathrm{C}-\mathrm{NMR}$ Spectrometry}

NMR spectra were acquired in $\mathrm{CDCl}_{3}$ at $25^{\circ} \mathrm{C}$ on a Bruker AVANCE $400-\mathrm{MHz}$ spectrometer equipped with a $5-\mathrm{mm}$ BBO probe. Chemical shifts $(\delta)$ are reported in ppm. ${ }^{1} \mathrm{H}-\mathrm{NMR}$ spectra were recorded with a relaxation delay of $1.0 \mathrm{~s}$ and an acquisition time of $4.09 \mathrm{~s}$. The acquisition parameters for ${ }^{13} \mathrm{C}-\mathrm{NMR}$ included a $90^{\circ}$ pulse width, a relaxation delay of $1.0 \mathrm{~s}$, and an acquisition time of $1.36 \mathrm{~s}$. Tetramethylsilane was used as a reference.

\section{Characterization by Matrix-Assisted Laser Desorption Ionization, Electrospray Ionization, and Atmospheric Pressure Chemical lonization Mass Spectrometry}

A Bruker 7T solariX Fourier-transform (FT) ion cyclotron resonance (ICR)-MS (Bruker, Bremen, Germany) was utilized for ultrahigh-resolution mass spectral analysis. The mass resolving power $(\mathrm{m} / \Delta \mathrm{m} 50 \%)$ was at least 100,000 at $\mathrm{m} / \mathrm{z} 400$ during calibration. For matrix-assisted laser desorption ionization (MALDI)-MS, the sample was dissolved in organic solvent (acetonitrile:water) at approximately $0.1 \mathrm{wt} \%$ concentration and spotted at $2 \mu \mathrm{l}$ on a ground stainless steel MTP 384 MALDI target plate (\#8280784, Bruker). Laser ionization was initiated with a Nd:YAG laser, $355 \mathrm{~nm}$, set between 12 and $60 \%$ relative laser power and $<50-\mu \mathrm{m}$ beam diameter. Approximately $2 \mu \mathrm{l}$ of $\alpha$-cyano-hydroxy-cinnamic acid
(HCCA) matrix was spotted on top of the dried surfactant sample prior to data collection. Mass spectra were collected in positive ion mode, and 50 time domain transient scans were co-added for each sample prior to fast FT and frequency to $\mathrm{m} / \mathrm{z}$ conversion. The mass spectrometer was internally calibrated to achieve subppm mass measurement accuracy on NaTFA in positive ion mode $(-0.086 \mathrm{ppm}$ at $\mathrm{m} / \mathrm{z} 702.863262 ; 0.138 \mathrm{ppm}$ at $\mathrm{m} / \mathrm{z}$ $1,246.762494$ ) and tuned for $\mathrm{m} / \mathrm{z} 100-1,500$ detection, at $0.7 \mathrm{~ms}$ ion time of flight in transfer optics.

For both electrospray ionization (ESI) and atmosphericpressure chemical ionization (APCI), 25 2.9-s time-domain signals were co-added. The unsaponifiables sample was dissolved in chloroform and diluted to $2 \mathrm{mg} / \mathrm{ml}$. The sample was further diluted to $10-20 \mu \mathrm{g} / \mathrm{ml}$ prior to mass spectral analysis. ESI was conducted in 90:10 methanol:chloroform with $1 \%$ formic acid and $100 \mu \mathrm{g} / \mathrm{ml}$ of sodium trifluoroacetate. The ion source was operated under the following conditions: ESI capillary at $4,500 \mathrm{~V}$, deflector plate at $-500 \mathrm{~V}$, nebulizer gas at $1 \mathrm{bar}$, dry temperature of $200^{\circ} \mathrm{C}$, and dry gas at $4 \mathrm{~L} / \mathrm{min}$. APCI was conducted in hexane with the following source conditions: corona discharge $4,000 \mathrm{~V}$, deflector plate $-500 \mathrm{~V}$, nebulizer gas 2 bar, vaporizer temperature $370^{\circ} \mathrm{C}$, dry gas $3.5 \mathrm{~L} / \mathrm{min}$, and dry temperature of $220^{\circ} \mathrm{C}$. The three most abundant sterol peaks $(\mathrm{m} / \mathrm{z}$ $=395.3669,397.3825$, and 383.3669) were mass-selected and fragmented prior to MS analysis. Fragmentation was conducted with collision induced dissociation (CID) in argon at $15-\mathrm{V}$ fragmentation energy. MS/MS results were compared to published literature and indicate the presence of stigmasterol, and campesterol. Peak lists were generated with a noise threshold of 10 standard deviations above the root mean square of the noise. Molecular formula assignments were generated with a custom 
script written in $\mathrm{R}$ programming language ( $\mathrm{R}$ Development Core Team, 2013). Plots of double bond equivalents (DBE) and mass spectra were also plotted in $\mathrm{R}$ with the use of the ggplot2 library.

\section{Differential Scanning Calorimetry}

Differential scanning calorimetry (DSC) was performed using a TA Instruments Q2000 DSC instrument calibrated with indium metal. Samples were analyzed by sealing them in aluminum samples pans and heating at $5^{\circ} \mathrm{C} / \mathrm{min}$ up to $60^{\circ} \mathrm{C}$, followed by cooling to $-20^{\circ} \mathrm{C}$ and then heating again to $60^{\circ} \mathrm{C}$.

\section{Determination of Hydrophilic-Lipophilic Balance}

The hydrophobic-lipophobic balance (HLB) is a measure of the degree to which a surfactant is hydrophilic or lipophilic. The HLB scale classifying surfactant function ranges between 0 and 18 , with $<6$ indicating a hydrophobic surfactant, 6-9 waterdispersible surfactants, and $>9$ as increasingly hydrophilic surfactant properties. HLB was determined by using a previously published method (Dehghan-Noudeh et al., 2005). A set of standard solutions with a known HLB was made from Span 80 , Tween 80 , and paraffin oil according to Supplementary Tables S4, S5. A sample containing paraffin oil and $0.5 \mathrm{wt} \%$ of each surfactant was also made and compared to the standard solutions. Each of the solutions was placed into a $15-\mathrm{ml}$ centrifuge tube and spun at room temperature for $4 \mathrm{~min}$ at $1,500 \mathrm{rpm}$. The tubes were removed, and the aqueous layer was measured using the graduations on the centrifuge tubes. The measurement was repeated four times for each solution and the average was calculated.

\section{RESULTS AND DISCUSSION}

\section{Extraction and Characterization of Algae Unsaponifiable Lipids}

To test the feasibility of creating a novel hydrophobic feedstock and characterize the hydrophobic donors for the renewable surfactant process, we extracted crude lipids in a processrelevant manner from algal biomass derived from the alga $S$. acutus. The biomass was subjected to a conversion process based on a dilute acid pretreatment followed by hexane-based lipid extraction, as described in the methods section (Dong et al., 2015). The lipids were subsequently saponified, creating a fattyacid-rich fraction for fuel synthesis ( $>90 \%$ of the lipids) and an unsaponifiables fraction ( $\sim \%$ of the lipids) containing the pigments; sterols; phytol; an aliphatic diterpene alcohol, usually bound to the hydrophilic headgroup of chlorophyll pigments; and other minor hydrocarbons. Three independent quantitative preparations of unsaponifiables from three respective biomass sources of $S$. acutus yielded $4.25 \pm 0.03 \%$ on a lipid basis. GC-MS analysis was able to discern at least seven different sterols in $S$. acutus biomass, with stigmasterol $(\Delta 7$ avenasterol) being the predominant species $(>34 \%$ of the total sterols), which together accounted for $35.7 \pm 0.8 \%$ of the unsaponifiables fraction. Phytol accounted for $33.5 \pm 1.4 \%$ of

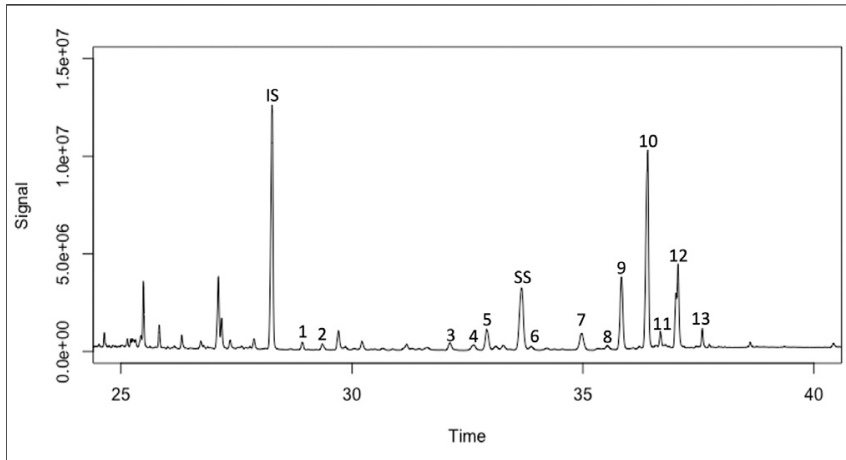

FIGURE 2 | Annotated gas chromatogram of sterols identified in Scenedesmus algal biomass; (1) MW 397, 28-carbon 4desmethylsterols- similar to ergosterol; (2) MW 411, 29-carbon 4desmethylsterols- triple unsaturated, (3) MW 401, 28-carbon 4desmethylsterols- similar to campesterol or ergosta-7-en-3 $\beta$-ol; (4) MW 397, 28-carbon 4-desmethylsterols- similar to ergosterol; (5) MW 413, 29-carbon 4-desmethylsterols- similar to stigmasterol, fucosterol, or $\Delta 5$ avenasterol; (6) MW 415, 29-carbon 4-desmethylsterols- similar to sitosterol or $\Delta 7$-stigmastenol; (7) MW 397, 28-carbon 4desmethylsterols- similar to ergosterol; (8) MW 415, 29-carbon 4desmethylsterols- similar to sitosterol or $\Delta 7$-stigmastenol; (9) MW 401, 28-carbon 4-desmethylsterols- similar to campesterol or ergosta-7-en33-ol; (10) MW 413, 29-carbon 4-desmethylsterols- similar to stigmasterol, fucosterol, or $\Delta 5$-avenasterol; (11) MW 401, 3 $\beta$ (trimethylsiloxy)-5.Xi.-Egost-7-ene; (12) MW 415, 29-carbon 4desmethylsterols- similar to sitosterol or $\Delta 7$-stigmastenol; (13) MW 415, $3 \beta$-(trimethylsiloxy)-5.Xi.-Stigmast-7-ene, IS = internal standard, cholestanol was included as an internal, non-native, standard for recovery calculations, and SS = surrogate standard, dihydrocholesterol.

the unsaponifiables, and thus together with sterols, represents $69.1 \pm 2.1 \%$ of the total fraction. A gas chromatogram is included to visualize the separation of the different sterols or general sterol categories for the predominant peaks identified (Figure 2).

To better understand the complex molecular composition of the unsaponifiable lipids from algae, we carried out a detailed high-resolution MS study by FT-ICR MS with both positive-ion ESI and APCI. In Figure 3, the mass spectra are shown alongside plots of DBE values against the number of carbon atoms, solely for the oxygenated species (those compounds containing between 1 and 3 oxygen atoms) identified in the ESI and APCI data. Here we see that by ESI (Figures $\mathbf{3 A}, \mathbf{C})$, phytol $\left(\mathrm{C}_{20}\right.$ and $\left.\mathrm{DBE}=1\right)$ is the most abundant signal observed, with smaller signals from oxygenated pigments (xanthophylls) and sterols. APCI (Figures 3B,D) reveals a more complete picture of sterol composition, with the most abundant peaks corresponding to stigmasterol, sitosterol, and campesterol. A total of 12 unique sterols were identified and confirmed by MS/MS analysis. In total, 12 sterols were unambiguously characterized with assigned formulae corresponding to known sterols (Table 1). Figures 3C,D shows the phytol, sterol, and pigment regions in green, blue, and red boxes, respectively, superimposed on plot of DBE vs. carbon number. The most abundant signals observed by ESI and APCI belong to these three classes; however, there are many compounds that fall outside of these structural boundaries. Compounds that likely contribute to other observed signals are aliphatic and cyclic alcohols, as well as phenols and 

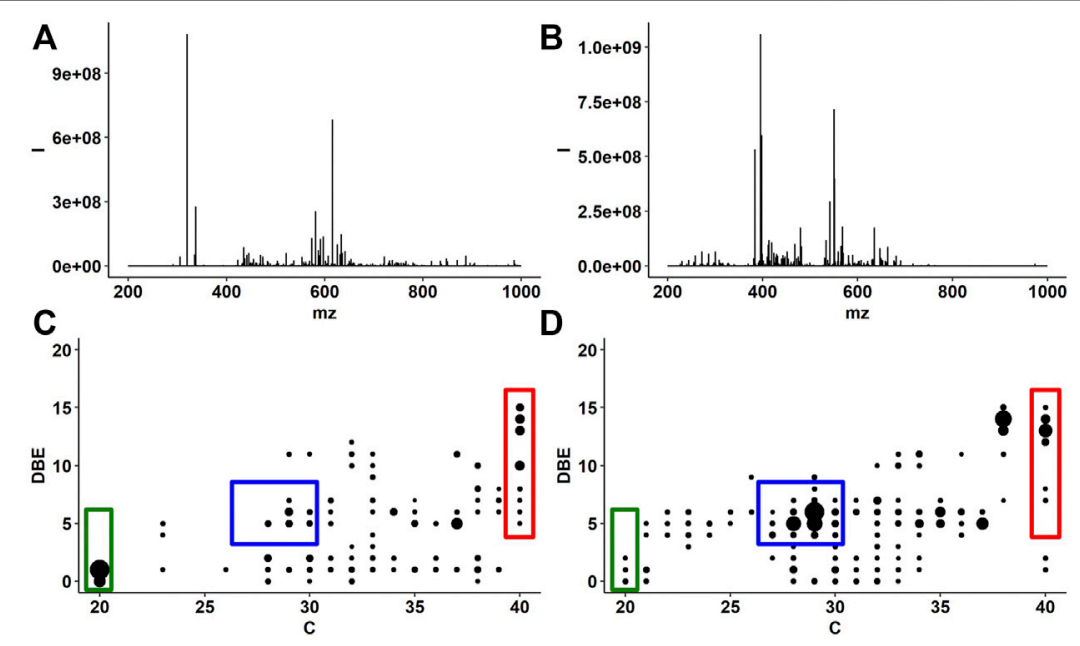

FIGURE 3 |FT-ICR mass spectra of the unsaponifiable compounds by ESI (A) and $\mathrm{APCl}$ (B). Plots of DBE vs. number of carbon atoms for the $\mathrm{O}_{1}-\mathrm{O}_{3}$-Containing compounds by ESI (C) and hydrocarbon- $\mathrm{O}_{2}$ compounds by $\mathrm{APCl}$ (D). lons observed in $\mathrm{APCl}$ are $\left[\mathrm{M}+\mathrm{H}-\mathrm{H}_{2} \mathrm{O}\right]^{+}$ions and were verified by analysis of sterol standards. Phytol, sterol, and pigment regions are indicated in green, blue, and red boxes, respectively, in (C) and (D).

TABLE 1 | Summary of identified and confirmed sterols in S. acutus unsaponifiable lipids, selected based on agreement with previously published literature Toivo et al. (2001), Fernandes (2007), Ryckebosch et al. (2014), Ahmed et al. (2015).

\begin{tabular}{|c|c|c|c|}
\hline Sterol ID & Exact Mass (neutral) & $\begin{array}{l}\text { Exact Mass (ion } \\
-\left[\mathrm{M}+\mathrm{H}-\mathrm{H}_{2} \mathrm{O}\right]^{+} \text {) }\end{array}$ & Elemental formula \\
\hline Desmosterol, 7-dehydrocholesterol & 384.339 & 367.3362603 & $\mathrm{C} 27 \mathrm{H} 44 \mathrm{O}$ \\
\hline Cholesterol & 386.355 & 369.3522603 & $\mathrm{C} 27 \mathrm{H} 46 \mathrm{O}$ \\
\hline Cholestanol $^{\mathrm{a}}$ & 388.371 & 371.3682603 & $\mathrm{C} 27 \mathrm{H} 48 \mathrm{O}$ \\
\hline Ergosterol, Coprostanol & 396.339 & 379.3362603 & $\mathrm{C} 28 \mathrm{H} 44 \mathrm{O}$ \\
\hline Brassicasterol & 398.355 & 381.3522603 & $\mathrm{C} 28 \mathrm{H} 46 \mathrm{O}$ \\
\hline Campesterol & 400.371 & 383.3682603 & $\mathrm{C} 28 \mathrm{H} 48 \mathrm{O}$ \\
\hline Campestanol & 402.386 & 385.3832603 & $\mathrm{C} 28 \mathrm{H} 50 \mathrm{O}$ \\
\hline Poriferasta-7,22,25-trienol & 410.355 & 393.3522603 & $\mathrm{C} 29 \mathrm{H} 46 \mathrm{O}$ \\
\hline Stigmasterol, $\Delta 5$-Avenasterol, Spinasterol, $\Delta 7$-Avenasterol & 412.371 & 395.3682603 & $\mathrm{C} 29 \mathrm{H} 48 \mathrm{O}$ \\
\hline Sitosterol & 414.386 & 397.3832603 & $\mathrm{C} 29 \mathrm{H} 50 \mathrm{O}$ \\
\hline$\Delta 7$-Stigmastenol & 416.402 & 399.3992603 & $\mathrm{C} 29 \mathrm{H} 52 \mathrm{O}$ \\
\hline Citrostadienol & 426.386 & 409.3832603 & $\mathrm{C} 30 \mathrm{H} 50 \mathrm{O}$ \\
\hline
\end{tabular}

${ }^{a}$ Cholestanol was included as an internal, non-native, standard.

polyphenols such as fat-soluble vitamins (Sánchez-Machado et al., 2002; Ryckebosch et al., 2014). These compounds likely have hydroxyl donors that also participate in the reactions that lead to incorporation with the hydrophilic polymer backbone, and will therefore contribute to the overall properties of the synthesized surfactants.

\section{Synthesis and Characterization of Succinic Anhydride-PEG Derivatives and Structural Confirmation}

Because the GC analysis indicated close to $70 \%$ of the unsaponifiables consisted of phytol and sterol derivatives, we utilized cholesterol and pure phytol isomers to test the synthesis and account for the surfactant properties from pure component synthesized products. The reaction scheme in
Figure 1 shows cholesterol as the hydrophobic moiety's hydroxyl donor, while phytol is expected to behave in the same manner by reacting the secondary alcohol for the sequential esterification with succinic anhydride. The unsaponifiables are complex, as described earlier, and will likely cause the heterogeneous incorporation of different donors, which will inevitably define the product quality and surfactant properties.

Synthesized surfactants and their intermediates derived from cholesterol, phytol, and unsaponifiables were extensively characterized by ${ }^{1} \mathrm{H}-\mathrm{NMR}$ and ${ }^{13} \mathrm{C}-\mathrm{NMR}$ to determine chemical structure and completeness of the reaction. The intermediate products were also characterized, and data are summarized in the supplemental information (Supplementary Figures S1-S5, with Supplementary Figures S1, S2 showing the ${ }^{1} \mathrm{H}-\mathrm{NMR}$ and Supplementary Figures S3-S5 the ${ }^{13} \mathrm{C}$-NMR data). 


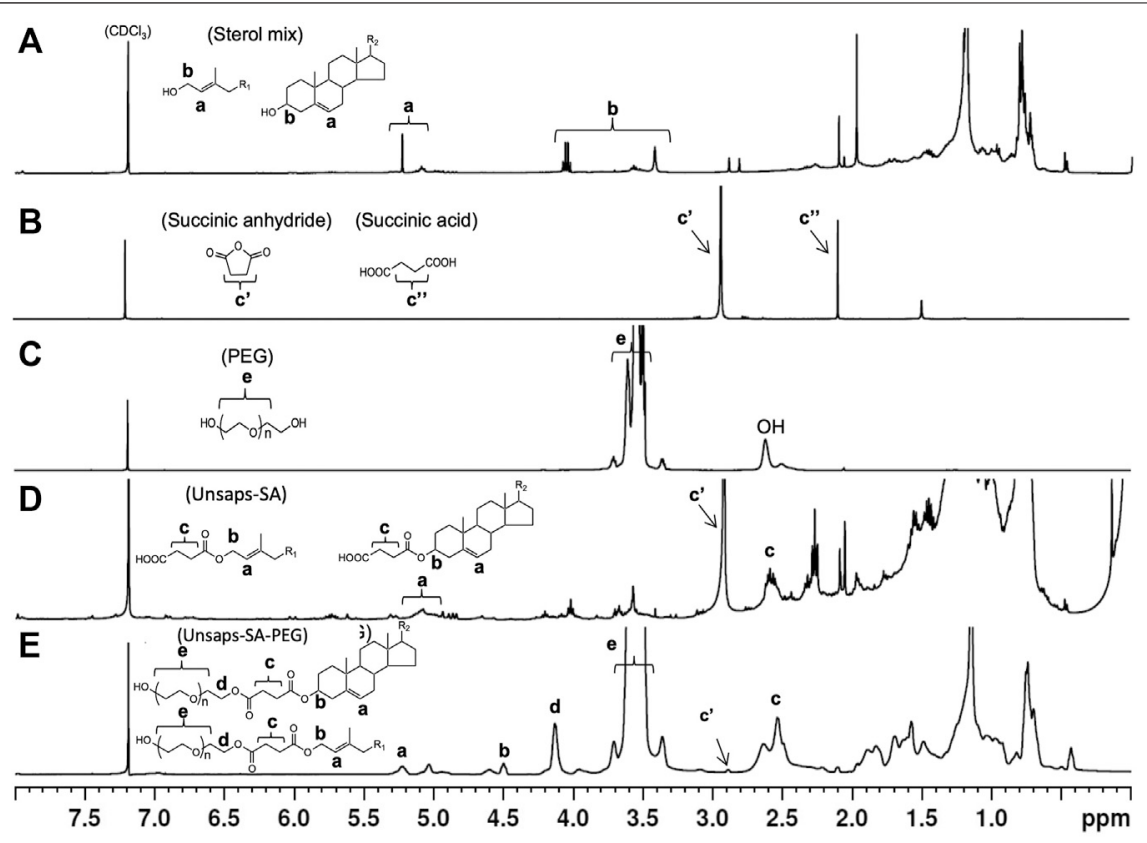

FIGURE 4 | ${ }^{1} \mathrm{H}-\mathrm{NMR}$ spectra of (A) sterol mixture, (B) succinic anhydride, (C) PEG, (D) unsaponifiables-SA, and (E) unsaponifiables-SA-PEG.

The unsaponifiables, subjected to an identical synthesis reaction as the pure component cholesterol and phytol products, produced an unsaponifiables-SA-PEG complex that shows the same functional groups incorporated. Figure 4 shows ${ }^{1} \mathrm{H}-\mathrm{NMR}$ spectra series from the sterol mixture. $\mathrm{H}_{\mathrm{a}}$ and $\mathrm{H}_{\mathrm{b}}$ in the extracted sterol mixture appear in the wide range of $4.98-5.28$ and $3.47-4.13$ ppm, respectively, suggesting that the sterol mixture contains both types of cholesterol and diterpene alcohol like phytol. As shown in Figure $4 \mathbf{E}$, in the reaction product of sterol mixture-succinate with PEG, $\mathrm{H}_{\mathrm{a}}$ and $\mathrm{H}_{\mathrm{b}}$ in sterol moiety appear at peak 5.28 and 4.55, respectively. Methylene protons in succinate moiety $\left(\mathrm{H}_{\mathrm{c}}\right)$ and in PEG moiety $\left(\mathrm{H}_{\mathrm{e}}\right)$ appear at 2.5-2.65 and 3.41-3.76 ppm, respectively. The most characteristic peak at $4.18 \mathrm{ppm}\left(\mathrm{H}_{\mathrm{d}}\right)$ is assigned to the terminal methylene proton of PEG coupled with sterol-SA. The chemical shifts of these peaks and spectrum pattern are consistent with those in cholesterolsuccinate-PEG and phytol-SA-PEG. All ${ }^{1} \mathrm{H}-\mathrm{NMR}$ data indicate that the obtained reaction product is an ester complex of primarily sterol and phytol-SA-PEG derivatives, and likely several uncharacterized hydroxylated compounds serving as additional reactants, which were found to be minor contributors in the mass spectrometry analysis. The intermediates and final products were also characterized by ${ }^{13} \mathrm{C}-\mathrm{NMR}$, and the data are included in supplemental information (Supplementary Figures S3-S5). The extensive NMR characterization supports completeness of the reaction and confirms the structure of the carboxyethyl-PEG- (or SA-PEG)-derived surfactants.

\section{Molecular Elucidation by MALDI-FT-ICR-MS}

To elucidate the incorporation of unsaponifiables into the surfactant, PEG, phytol-SA-PEG, cholesterol-SA-PEG, and the
unsaponifiables-SA-PEG were analyzed by MALDI FT-ICR MS. Figure 5 shows the broadband mass spectra from the four samples. We observe a shift to greater $\mathrm{m} / \mathrm{z}$ values with derivatization of the lipid-derived tails, which is due to the incorporation of the hydrophobic moieties (cholesterol, phytol, and other unsaponifiables). A complete list of observed ions for phytol-succinate-PEG and cholesterol-succinate-PEG is provided as supplemental information (Supplementary Tables S1, S.2). These spectra confirm that phytol and cholesterol incorporate into the PEG-succinate head, as shown by NMR. By comparison, we see that the spectrum for the unsaponifiables-SA-PEG is far more complex than the phytol and cholesterol counterparts. This indicates a heterogeneous incorporation of hydrophobic moieties from the unsaponifiable sample. This is not surprising, considering the diversity of compounds observed by ESI- and APCI-FT-ICR MS. Despite the complexity of the unsaponifiables, the polydispersity $\left(\mathrm{M}_{\mathrm{n}} / \mathrm{M}_{\mathrm{w}}\right)$ of the resultant surfactant polymer is not much greater than the PEG 1000 used for synthesis, indicating a relatively narrow range of synthesis products. The data obtained by mass spectrometry were confirmed by size exclusion chromatography, with a similar molecular weight distribution (Supplementary Figure S6; Supplementary Table S3).

By MALDI-MS, we were able to identify both the phytoland stigmasterol-functionalized PEG-SA surfactants in the unsaponifiable-reacted surfactant sample. However, the relative abundance for the phytol-SA-PEG was surprisingly low, given its high concentration in the starting sample. Likewise, we were unable to adequately identify other sterol-SA-PEG signals, which indicates that other compounds in the unsaponifiable sample may be incorporating into the surfactant with greater efficiency 


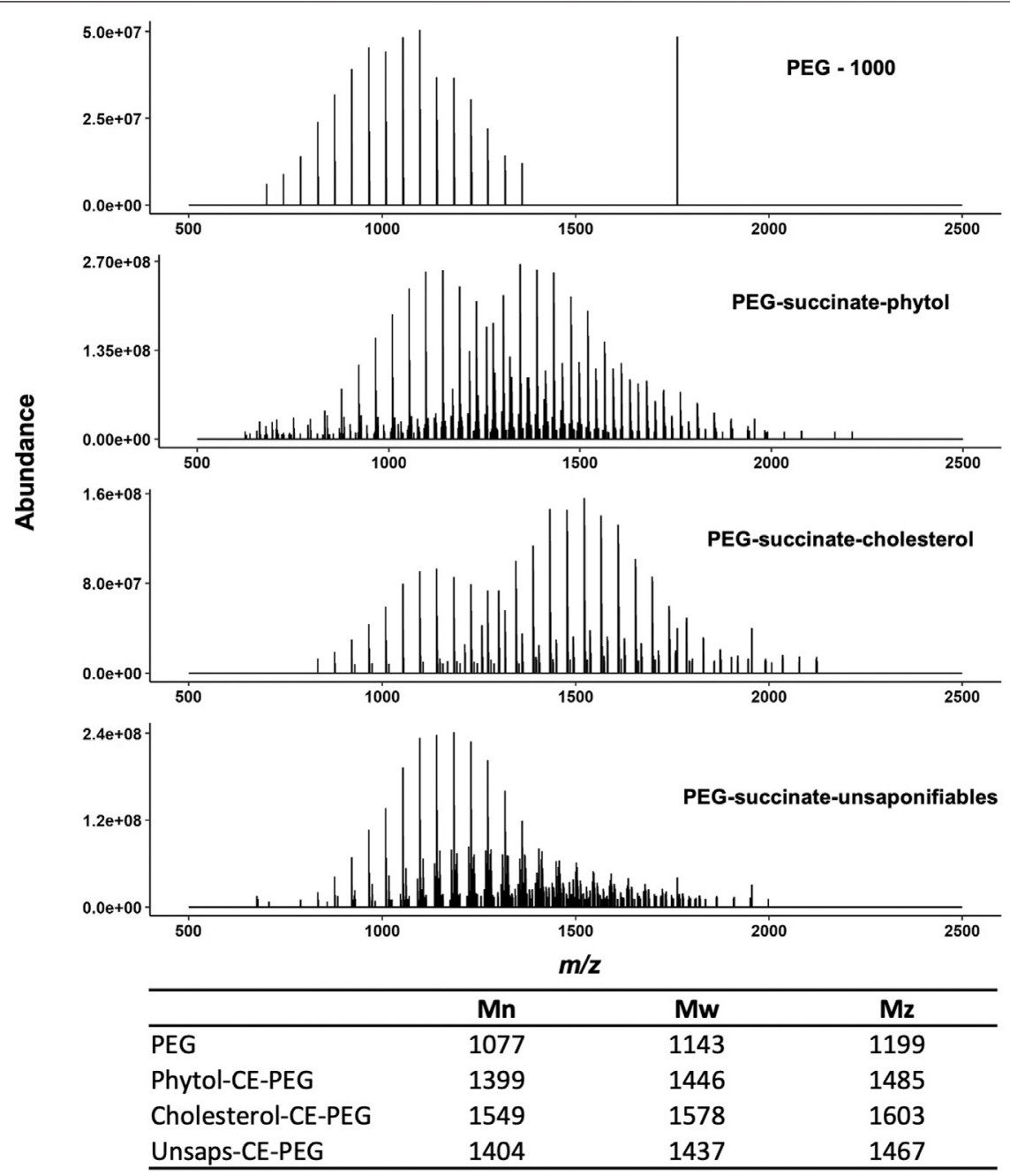

FIGURE 5 | Summary of MALDI-FT-ICR-MS of surfactants synthesized from phytol, cholesterol, and S. acutus unsaponifiables. Top to bottom are PEG 1000, phytol-SA-PEG, cholesterol-SA-PEG, and unsaponifiables-SA-PEG. Bimodal distributions are observed in the reacted surfactant samples, where the lower $\mathrm{m} / \mathrm{z}$ distribution corresponds to unreacted PEG 1000. The respective calculated molecular weight distribution is included; $\mathrm{Mn}=\mathrm{number}$ average, $\mathrm{Mw}=\mathrm{weight}$ average, and $\mathrm{Mz}=\mathrm{Z}$ average.

than the sterols and phytol. This may indicate the presence of more reactive phenolic or polyphenolic compounds within the unsaponifiable sample. Alternatively, the ionization efficiency of the phytol and sterol-SA-PEG may be lower than that of other products, making them underrepresented in the mass spectrum. Future work is needed to better understand the heterogeneous incorporation of mixed samples from algae lipids.

\section{DSC Analysis}

DSC, based on a heat-cool-heat cycle, was used to look for any polymorphic behavior of the material, as well as to erase the thermal history of the sample on the first heat. For the heat-coolheat cycles, no polymorphism was noted, and the melting point overlaid on both heat cycles, indicating a stable crystal structure. Figure 6 contains the thermograms for all three products. The melting points of all three are similar, and as would be expected, the melting of the mixed product falls between that of cholesterol and phytol, which is consistent with the respective composition of the unsaponifiables fraction. The calculated melting point for cholesterol-SA-PEG product was $41.1^{\circ} \mathrm{C}$, for phytol-SA-PEG $34.2^{\circ} \mathrm{C}$, and for the unsaponifiables-SA-PEG $39.3^{\circ} \mathrm{C}$. The intermediate melting temperature for the unsaponifiable lipid derivatives makes sense, as this was a mixture of different components that contribute to the final product properties. This also supports the concept that the respective composition of the hydrophobic moieties will be a determining factor toward the final surfactant properties.

\section{Surfactant Properties by HLB Measurements}

HLB measurements were selected as the primary surfactant property metric that can help identify the suitability of the 


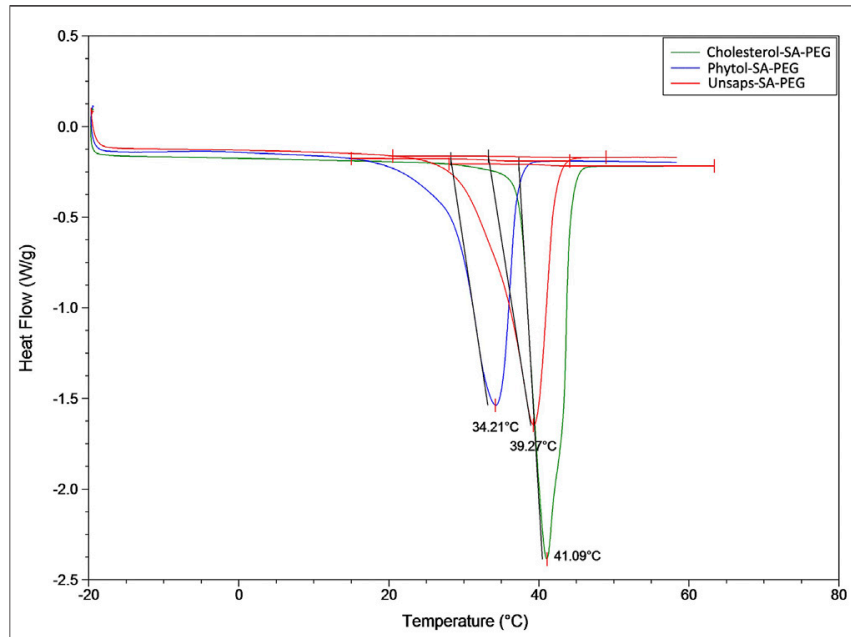

FIGURE 6 | Differential scanning calorimetry profiles of each of the final products: carboxyethyl esters of cholesterol, phytol, and unsaponifiable surfactant derivatives, shown as succinic anhydride-PEG esters.

novel surfactant. This measurement gives information on the relative solubility of oil-in-water emulsions, and the value, though approximate, gives a general idea on the type of surfactant applications that could be met with the products. Figure 7 shows a photo of the centrifuge tubes for the samples as a visual comparison. As described for the DSC measurements, the respective properties for the phytol and cholesterol derivatives are predictive of the properties of the unsaponifiables surfactant mixtures. The HLB of the algaederived mixed 1000 PEG surfactant was the same as phytol, which came in just above standard \#4, having an HLB value of 9.65. This indicates that a water-oil emulsifier that is watersoluble was produced with reasonable detergency. For comparison, the linear alkyl benzene sulfonate dodecylbenzenesulfonic acid has an HLB value of 11.7 (chemical database reference CAS 69669-44-9). Surfactants in this range can typically be used in cosmetic, food, and pharmaceutical applications. If a slightly different HLB value is desired, the PEG chain length could be modified. Also, this surfactant could be blended with another surfactant with a known HLB to produce a final surfactant blend with the exact HLB desired.

\section{CONCLUSION}

We demonstrate here for the first time the synthesis of renewable surfactants from the algae unsaponifiable lipid fraction, using an alternative, green, and less reactive (flammable and explosive) chemical approach to ethoxylation. The synthesis reaction is based on the sequential esterification of succinic anhydride and polyethylene glycol of the hydroxyl functional groups of novel hydrophobic moieties. The valorization of the unsaponifiable fraction, isolated from an algae lipids process stream, is an effective route to support the overall biorefinery process

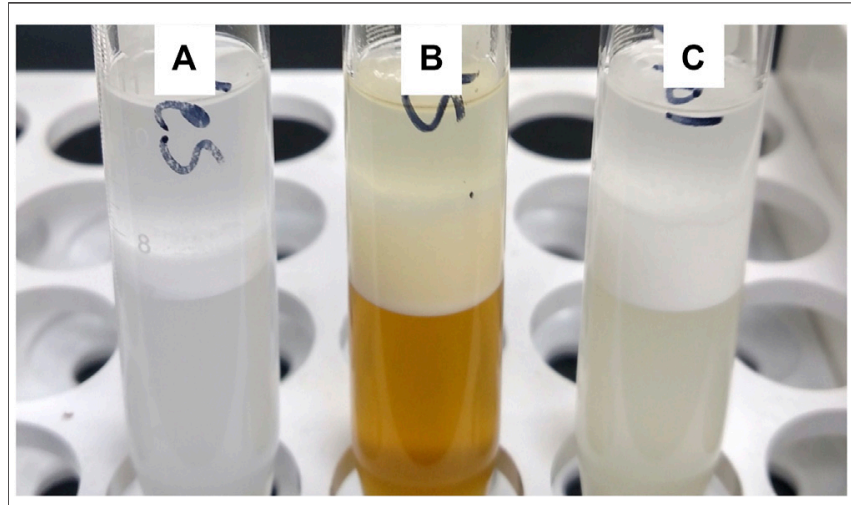

FIGURE 7 | Visualization of (A) HLB measurement of cholesterol-SA-PEG, (B) unsaponifiables-SA-PEG, and (C) phytol-SA-PEG.

economics, while simultaneously improving the hydrotreating properties of the remaining fatty acid stream toward biofuel production. Although reagents and solvents used in this initial synthesis were primarily petroleum-derived, future iterations of this process could incorporate bio-derived components, such as succinate produced by fermentation of algal sugars from combined algal processing, to further reduce fossil carbon utilization, or overall carbon intensity. Future research should focus on life cycle analyses to identify areas where carbon intensity of surfactant synthesis can be further reduced.

The unsaponifiable lipid products were characterized in great detail based on ultrahigh-resolution mass spectrometry, which revealed a unique and highly complex mixture of hydrophobic donors, primarily derived from the diterpenoid hydrocarbon biosynthetic pathways to the surfactant synthesis reaction chemistry. This in-depth profiling supports the surfactant property characterization and the identified carboxy-ethyl esterified products by NMR. Although the resulting surfactants are mixed components, from the detailed molecular and spectroscopic profiling it appears that the respective molecular composition of the sterols does not determine surfactant properties, and the primary determination of properties may be the balance between the bulk constituents: sterols and phytols. A more detailed study of the respective synthetic reaction rates of the individual components in the unsaponifiable lipids and the utilization of additional surfactant hydrophilic donors is ongoing in our laboratory and will be reported on in future manuscripts.

\section{DATA AVAILABILITY STATEMENT}

The raw data supporting the conclusion of this article will be made available by the authors, without undue reservation.

\section{AUTHOR CONTRIBUTIONS}

LL, GF, EC, TD, and PP contributed to the design of the study. GF carried out all chemical synthesis reactions and hydrophilic 
lipophilic balance analysis, SVW performed the chemical extraction and analysis of the unsaponifiable lipids, TD contributed to the extraction of lipids and unsaponifiables, SR performed the high-resolution mass spectrometry analysis, RK performed NMR analysis, and EC performed size exclusion chromatography and differential scanning calorimetry. GF and LL wrote the first draft of the manuscript. EC, SR, SVW, and TD wrote sections of the manuscript. All authors contributed to manuscript revision and read and approved the submitted version. LL secured funding and conceived the original concept of algae lipid valorization to biobased products.

\section{FUNDING}

This work was authored by the National Renewable Energy Laboratory, operated by Alliance for Sustainable Energy, LLC, for the United States Department of Energy (DOE) under Contract No. DE-AC36-08GO28308. Funding provided by the United States Department of Energy Office of Energy Efficiency and Renewable Energy Bioenergy Technologies Office, WBS

\section{REFERENCES}

Ahmed, F., Zhou, W., and Schenk, P. M. (2015). Pavlova Lutheri Is a HighLevel Producer of Phytosterols. Algal Res. 10, 210-217. doi:10.1016/ j.algal.2015.05.013

American Oil Chemists' Society (2009). "AOCS Official Method Ca 6b-53," in Unsaponifiable Matter (Urbana, IL: AOCS Press, Wiley).

Ashford, R. D. (2011). Ashford's Dictionary of Industrial Chemicals. Third Edition. Cornwall, England: Wavelength Publications.

Davis, R., Kinchin, C. M., Markham, J., Tan, E. C. D., Laurens, L. M. L., Sexton, D., et al. (2014). Process Design and Economics for the Conversion of Algal Biomass to Biofuels: Algal Biomass Fractionation to Lipid- and Carbohydrate-Derived Fuel Products. Golden, CO: National Renewable Energy Laboratory.

Dehghan-Noude, G., Housaindokht, M., and Bazzaz, B. S. (2005). Isolation, Characterization, and Investigation of Surface and Hemolytic Activities of a Lipopeptide Biosurfactant Produced by Bacillus Subtilis ATCC 6633. J. Microbiol. 43, 272-276.

Dong, T., Knoshaug, E. P., Davis, R., Laurens, L. M. L., Van Wychen, S., Pienkos, P. T., et al. (2016). Combined Algal Processing: A Novel Integrated Biorefinery Process to Produce Algal Biofuels and Bioproducts. Algal Res. 19, 316-323. doi:10.1016/j.algal.2015.12.021

Fernandes, P., and Cabral, J. M. S. (2007). Phytosterols: Applications and Recovery Methods. Bioresour. Techn. 98, 2335-2350. doi:10.1016/ j.biortech.2006.10.006

Folmer, B. M. (2003). Sterol Surfactants: from Synthesis to Applications. Adv. Colloid Interf. Sci. 103, 99-119. doi:10.1016/s0001-8686(01)00100-2

Johansson, I., and Svensson, M. (2001). Surfactants Based on Fatty Acids and Other Natural Hydrophobes. Curr. Opin. Colloid Interf. Sci. 6, 178-188. doi:10.1016/s1359-0294(01)00076-0

Kjellin, M., and Johansson, I. (2010). Surfactants from Renewable Resources. Hoboken, NJ: John Wiley \& Sons. doi:10.1002/9780470686607

Koutinas, A. A., Chatzifragkou, A., Kopsahelis, N., Papanikolaou, S., and Kookos, I. K. (2014). Design and Techno-Economic Evaluation of Microbial Oil Production as a Renewable Resource for Biodiesel and Oleochemical Production. Fuel 116, 566-577. doi:10.1016/ j.fuel.2013.08.045
1.3.2.001. The views expressed in the article do not necessarily represent the views of the DOE or the United States Government. The United States Government retains and the publisher, by accepting the article for publication, acknowledges that the United States Government retains a nonexclusive, paid-up, irrevocable, worldwide license to publish or reproduce the published form of this work, or allow others to do so, for United States Government purposes.

\section{ACKNOWLEDGMENTS}

We are grateful to Paris Spinelli for providing the extracted lipids and unsaponifiable fraction from the algae and to Oliver Palardy for establishing the original GC/MS methodology.

\section{SUPPLEMENTARY MATERIAL}

The Supplementary Material for this article can be found online at: https://www.frontiersin.org/articles/10.3389/fceng.2021.768382/ full\#supplementary-material

Kruger, J. S., Christensen, E. D., Dong, T., Van Wychen, S., Fioroni, G. M., Pienkos, P. T., et al. (2017). Bleaching and Hydroprocessing of Algal Biomass-Derived Lipids to Produce Renewable Diesel Fuel. Energy Fuels 31, 10946-10953. doi:10.1021/acs.energyfuels.7b01867

Laurens, L. M. L., Van Wychen, S., McAllister, J. P., Arrowsmith, S., Dempster, T. A., McGowen, J., et al. (2014). Strain, Biochemistry, and Cultivationdependent Measurement Variability of Algal Biomass Composition. Anal. Biochem. 452, 86-95. doi:10.1016/j.ab.2014.02.009

Laurens, L. M. L., Nagle, N., Davis, R., Sweeney, N., Van Wychen, S., Lowell, A., et al. (2015). Acid-catalyzed Algal Biomass Pretreatment for Integrated Lipid and Carbohydrate-Based Biofuels Production. Green. Chem. 17, 1145-1158. doi:10.1039/c4gc01612b

Laurens, L. M. L., Markham, J., Templeton, D. W., Christensen, E. D., Van Wychen, S., Vadelius, E. W., et al. (2017). Development of Algae Biorefinery Concepts for Biofuels and Bioproducts; a Perspective on Process-Compatible Products and Their Impact on Cost-Reduction. Energy Environ. Sci. 10, 1716-1738. doi:10.1039/c7ee01306j

Lim, J. C., and Chung, D.-W. (2011). Study on the Synthesis and Characterization of Surface Activities of Hydrophilic Derivatives of $\beta$ sitosterol. J. Appl. Polym. Sci. 125, 888-895. doi:10.1002/app.36259

Pleissner, D., Lau, K. Y., Zhang, C., Lin, C. S. K., and Lin, K. (2014). Plasticizer and Surfactant Formation from Food-Waste- and Algal Biomass-Derived Lipids. ChemSusChem 8, 1686-1691. (in press). doi:10.1002/ cssc. 201402888

R Development Core Team (2013). R: A Language and Environment for Statistical Computing. Vienna, Austria: R Foundation for Statistical Computing. Available at: http://www.R-project.org.

Ryckebosch, E., Bruneel, C., Termote-Verhalle, R., Muylaert, K., and Foubert, I. (2014). Influence of Extraction Solvent System on Extractability of Lipid Components from Different Microalgae Species. Algal Res. 3, 36-43. doi:10.1016/j.algal.2013.11.001

Sánchez-Machado, D. I., López-Hernández, J., and Paseiro-Losada, P. (2002). High-performance Liquid Chromatographic Determination of AlphaTocopherol in Macroalgae. J. Chromatogr. A. 976, 277-284. doi:10.1016/ s0021-9673(02)00934-2

Toivo, J., Phillips, K., Lampi, A.-M., and Piironen, V. (2001). Determination of Sterols in Foods: Recovery of Free, Esterified, and Glycosidic Sterols. J. Food Compost. Anal. 14, 631-643. doi:10.1006/jfca.2001.1019 
Urbin, S., Scheibel, J. J., Vinson, P. K., Depa, P. K., Reilman, R. T., and Steffey, M. P. (2014). Detergent Compositions Comprising Sustainable Surfactant Systems Comprising Isoprenoid-Derived Surfactants. U.S. Patent and Trademark Office, 20140148375 A1.

Wagner, J. L., Ting, V. P., and Chuck, C. J. (2014). Catalytic Cracking of Sterol-Rich Yeast Lipid. Fuel 130, 315-323. doi:10.1016/j.fuel.2014.04.048

Conflict of Interest: The authors declare that the research was conducted in the absence of any commercial or financial relationships that could be construed as a potential conflict of interest.

Publisher's Note: All claims expressed in this article are solely those of the authors and do not necessarily represent those of their affiliated organizations, or those of the publisher, the editors and the reviewers. Any product that may be evaluated in this article, or claim that may be made by its manufacturer, is not guaranteed or endorsed by the publisher.

Copyright $\odot 2022$ Fioroni, Katahira, Van Wychen, Rowland, Christensen, Dong, Pienkos and Laurens. This is an open-access article distributed under the terms of the Creative Commons Attribution License (CC BY). The use, distribution or reproduction in other forums is permitted, provided the original author(s) and the copyright owner(s) are credited and that the original publication in this journal is cited, in accordance with accepted academic practice. No use, distribution or reproduction is permitted which does not comply with these terms. 